\title{
Gclc deletion in surface-ectoderm tissues induces microphthalmia
}

Brian Thompson ${ }^{1}$, Ying Chen ${ }^{1}$, Julien Philippe ${ }^{2}$, David Anderson ${ }^{3}$, Jaya Prakash Golla ${ }^{1}$, Emily Davidson ${ }^{1}$, Nicholas Apostolopoulos ${ }^{4}$, Kevin Schey ${ }^{3}$, Nicholas Katsanis ${ }^{2}$, David J. Orlicky ${ }^{5}$, David Thompson ${ }^{6}$, Vasilis Vasiliou $^{1 *}$

1 Department of Environmental Health Sciences, Yale School of Public Health, Yale University, New Haven, CT, USA.

2 Center for Human Disease Modeling, Duke University Medical Center, Durham, NC, USA.

3 Department of Biochemistry and Mass Spectrometry Research Center, Vanderbilt University School of Medicine, Nashville, TN, USA.

4 Department of Ophthalmology \& Visual Science, Yale School of Medicine, New Haven, CT, USA.

5 Department of Pathology, School of Medicine, University of Colorado, Aurora, CO, USA.

6 Department of Clinical Pharmacy, Skaggs School of Pharmacy, University of Colorado, Aurora, CO, USA.

Corresponding author: Vasilis.vasiliou@yale.edu

\section{Abstract}

Glutamate cysteine ligase catalytic subunit (Gclc) is the catalytic subunit for the glutamate-cysteine ligase (Gcl) enzyme. Gcl catalyzes the rate limiting step in glutathione (GSH) synthesis. Gclc is highly expressed in the developing eye. To define the regulatory role of $\mathrm{Gclc}$ in eye development, we developed a novel, Le-Cre transgene-driven, Gclc knockout mouse model. Gclc ${ }^{f / f} / L e-C r e^{T g /-}$ mice present with deformation of the retina, cornea, iris, and lens, consistent with a microphthalmia phenotype. Controlling for the microphthalmia phenotype of $G c l c^{w t / w t} / L e-C r e^{T g /-}$ mice revealed that $G c l c^{f / f} / L e-C r e^{T g /-}$ mice have a more severe microphthalmia phenotype. Thus, the loss of Gclc expression exacerbates the microphthalmia phenotype in Le-Cre mice. $G c l c^{f f f} / L e-C r e^{T g /-}$ eyes present with reduced retinal and lens epithelium proliferation and increased lens cell death. Imaging mass spectrometry of ocular tissues revealed changes in the intensity and distribution of several lipid species and proteins in the retina and corneas of $\mathrm{Gclc} / \mathrm{flf} / \mathrm{Le}-\mathrm{Cr} \mathrm{e}^{\mathrm{Tg} /}$ - eyes. Lastly, using splice-blocking morpholinos and CRISPR/Cas9, we created two gclc knockdown zebrafish models, both of which display a microphthalmia phenotype. Combined, the mouse and zebrafish results indicate that, in chordates, Gclc has a conserved role in regulating eye development. In summary, these novel animal models are useful tools for elucidating the mechanisms involved in microphthalmia development.

\section{Introduction}

Microphthalmia is a debilitating congenital malformation that affects around 1 in 7000 births [1-3]. In individuals with microphthalmia, one (unilateral) or both (bilateral) eyes are abnormally small (e.g., in adults, a corneal diameter less than $10 \mathrm{~mm}$ and an antero-posterior diameter of the globe less than $20 \mathrm{~mm}$ [4]). Microphthalmia can be classified into two main categories: total microphthalmia or partial microphthalmia [5]. In total microphthalmia, both the anterior and posterior segments are shortened. In contrast, only either the anterior or the posterior segment are abnormally small in partial microphthalmia. In partial microphthalmia cases where the anterior segment is abnormally small, lens development is often impaired. Lens development is regulated by a myriad of extracellular signaling pathways and gene regulatory networks (reviewed here [6]). Approximately $80 \%$ of microphthalmia cases can be explained by pathogenic mutations in $S O X 2, O T X 2, P A X 6$, VSX2, RAX, FOXE3, STRA6, ALDH1A3, or RARB [3, 7]. The remaining $20 \%$ of microphthalmia cases may be explained by exposure to environmental factors, such as pharmaceuticals (e.g, thalidomide [8]), ionizing radiation exposure [9, 10], fungicide exposure (e.g., benomyl [11]) and gestationally-acquired infections [12], or other factors such as maternal vitamin A deficiency [13, 14]. Blindness or limited vision is observed in individuals with the severest forms of microphthalmia. Indeed, microphthalmia is a major cause of childhood blindness, accounting for $\approx 11 \%$ of the cases [1]. Currently, there is no cure for the severe loss of vision associated with microphthalmia. While surgical treatments exist, they only address the cosmetic abnormalities associated with microphthalmia [15]. However, recent research demonstrating the efficacy of nonsense 
suppression (inhibiting the effect of a nonsense mutation by pharmacologically increasing the likelihood of a near cognate aminoacyl-tRNA substitution) to rescue lens development when administered postnatally in a Pax6-deficient mouse model has provided hope that treatments for microphthalmia can be developed [16]. Clearly, a more complete understanding of the mechanisms regulating lens development should accelerate the discovery of preventative strategies and/or novel therapeutic treatments for microphthalmia.

Glutathione (GSH), an intracellular antioxidant, is highly abundant in the eye, with concentrations of $20 \mathrm{mM}$ or more occurring in the lens cortex and epithelium [17, 18]. It is important for physiological detoxification of electrophiles and oxidants in the lens cortex and epithelium [19]. Through these functions, GSH maintains lens clarity and, as such, a decline in GSH levels is associated with cataract development [20, 21]. GSH biosynthesis involves a two-step enzymatic process [22] involving glutamate-cysteine ligase (GCL) followed by glutathione synthase (GSS). GCL, the rate limiting step in GSH synthesis, comprises a catalytic subunit (GCLC) and a modifier subunit (GCLM). Impaired GCLC or GCLM function limits GSH synthesis and reduces intracellular GSH concentrations. Thus, several Gclm [23] and Gclc [24-26] -deficient mouse models have been created to explore the role of GSH in physiological and pathophysiological conditions. For example, such mouse models have identified a role for GSH in protection against steatosis and neurodegeneration [24, 27]. A Lens Glutathione Synthesis KnockOut (LEGSKO) mouse model revealed suppression of Gclc expression induced age-related nuclear cataract development [26]. In this model, Gclc deletion occurs in the lens at embryonic day (E) 10.5 [26]. However, the role of Gclc and GSH in the early stages of lens development (i.e., prior to E10.5) remains uncharacterized.

We have developed novel mouse and zebrafish models in which GSH synthesis is reduced early in lens development. This was accomplished by genetically ablating Gclc in the surface-ectoderm derived tissues (i.e., cornea, lens, conjunctiva, eyelid) by cross-breeding Gclc floxed mice ( $\left.G c l c^{f l f l}\right)$ mice [24] with mice hemizygous for the Le-Cre transgene [28]. The Le-Cre transgene utilizes the Pax6-PO promoter to limit ocular Cre expression to only the lens, conjunctiva, and cornea starting at E9.5 [28]. In addition, since GSH is abundant in zebrafish [29] and gclc is highly expressed in the developing zebrafish eye [30], we postulated that it has a conserved role in regulating zebrafish eye development. To evaluate this possibility, we developed novel gclcsuppressed zebrafish models using splice-blocking morpholinos or CRISPR/Cas9. Herein, we present the phenotypic characterization of targeted GSH synthesis reduction in surface ectoderm-derived tissues during early mouse and fish eye development.

\section{Methods}

\section{Generation of Surface-Ectoderm Specific Gclc Knock-out Mice}

In order to delete Gclc from surface ectoderm-derived tissues, Gclc floxed $\left(G c l c^{f / f}\right)$ mice $(\mathrm{C} 57 \mathrm{BL} / 6 \mathrm{~J}$ background) were bred with $L e-C r e\left(\mathrm{FVB} / \mathrm{N}\right.$ background) transgenic mice $\left(L e-C r e^{T g /-}\right)$. The resultant Gclc $c^{f / f} / L e-$ $C r e^{T g /-}(G c l c \mathrm{KO})$ mice have ocular Cre recombinase expression restricted to only the conjunctiva, cornea, eyelid and lens from embryonic day 9 (E9) [28]. The generation of the Le-Cre (Tg(Pax6-cre,GFP)1Pgr) transgenic and $G c l c^{f / f}$ mice have been previously described [24, 29]. These mice [28] were originally produced by Dr. Ruth Ashery-Padan (Department of Human Molecular Genetics and Biochemistry, Sackler Faculty of Medicine, Tel Aviv University), and were obtained from Dr. David Beebe (Department of Ophthalmology and Visual Sciences, Washington University School of Medicine). Gclc HET were continuously crossed with Gclc/f mice to convert their genomic background towards $\mathrm{C} 57 \mathrm{BL} / 6 \mathrm{~J}$ and generate the three experimental mouse

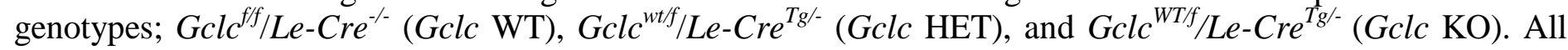
data presented in this paper was generated from B6/FVB mixed background mice. Mice were group-housed and maintained on 12-hour light-dark cycles, with food and water available ad libitum. All experiments were performed in strict accordance with the National Institutes of Health guidelines, with protocols approved by the Yale University Institutional Animal Care and Use Committee. 


\section{Generation of Le-Cre Transgene Control Mice}

To generate Le-Cre transgene control mice, we bred Gclc $c^{f / f} / L e-C r e^{-/-}\left(G c l c\right.$ WT) mice with $G c l c^{w t / f} ; L e-C r e^{T g /-}$ (Gclc HET) mice. The F1 mice (Gclc $c^{w t f} / L e-C r e^{T g /-}$ and $\left.G c l c^{w t f} / L e-C r e^{-/-}\right)$were then interbred to produce F2 mice of the $\mathrm{Gclc} \mathrm{c}^{w t / w t} / \mathrm{Le}-\mathrm{Cr} e^{T g /-}$ (WT/Cre) genotype. These WT/Cre mice served as Le-Cre transgene control mice.

\section{Genotyping}

Genomic DNA, obtained from a $2 \mathrm{~mm}$ ear punch collected at post-natal day (P) 14, was extracted using DirectPCR Lysis Reagent (Tail) (Viagen Biotech, Los Angeles, CA) and PCR Grade Proteinase K (SigmaAldrich) according to the manufacturer's protocol. Briefly, the ear punch sample was placed in $100 \mu \mathrm{L}$ DirectPCR Lysis Reagent (Tail) containing $1 \mu \mathrm{L}$ PCR Grade Proteinase K. The sample was incubated overnight at $55^{\circ} \mathrm{C}$, and subsequently incubated at $85^{\circ} \mathrm{C}$ for 45 minutes. Two separate PCR reactions were needed to properly describe each individual animal: one detected the $G c l c(w t)$ and $C r e(T g)$ alleles (left lane), and the other detected the Gclc(f) allele (right lane). Gclc alleles were determined using 5'-CTATAATGTCCTGCACTGGG and 5'-TAGTGAACGCTGTTAAAGG and 5'-CGGGTGTTGGGTCGTTTGT and the presence of the Cre transgene as identified using 5'-GCGGTCTGGCAGTAAAAACTATC and 5'-GTGAAACAGCATTGCCACTT, as previously described [24].

\section{Histopathological Examination and Immunohistochemical Staining}

E14.5 mice were collected, genotyped, and fixed overnight in 10\% neutral buffered formalin (Sigma-Aldrich). Ocular tissues were processed (i.e., dehydrated) and embedded in paraffin wax and sectioned (5 $\mu$ m thickness) onto slides by Dr. Mark Petrash (University of Colorado School of Medicine). A section from both eyes of each mouse was stained with hematoxylin and eosin (H\&E). Immunostaining was conducted using the TSA Plus Biotin Kit (Perkin Elmer, Waltham, MA) according to the manufacturer's protocol. Briefly, slides were blocked with TNB Blocking Buffer (Perkin Elmer, Waltham, MA) for 30 minutes at room temperature. Subsequently, slides were incubated overnight with primary antibody in TNB at $4{ }^{\circ} \mathrm{C}$. After primary antibody exposure, the slides were washed 3 times for 5 minutes with TNT buffer. Subsequently, the slides were incubated with horseradish peroxidase (HRP) conjugated secondary antibody for 30 minutes at room temperature. Slides were then washed 3 times for 5 minutes with PBST (0.05\% Tween-20, 1 X PBS) at room temperature. Next, the slides were incubated with TSA Plus working solution (Perkin Elmer, Waltham, MA) for 10 minutes at room temperature. The slides were subsequently incubated in streptavidin conjugated (SA)-HRP (diluted 1:100 in TNB) for 30 minutes at room temperature, Harris hematoxylin counterstain was applied, and mounted for imaging. Antibodies: PAX6 (Abcam, ab195045), GCLC (Abcam, ab53179), Ki67 (Abcam, ab21700), $\beta$ Catenin (Abcam, ab32572), and Rabbit IgG H\&L HRP (Abcam, ab6721). All antibodies were used at a concentration of 1:200 unless otherwise stated. TUNEL staining was performed by the Yale University Pathology Tissue Services.

\section{Quantification of GSH and GSSG}

To quantify GSH and GSSG, postnatal day (P) 21 Gclc KO and Gclc WT mice were anesthetized and eyes enucleated and immediately assayed using the GSH/GSSG Ratio Detection Assay Kit (Fluorometric-Green) (Abcam) according to the manufacturer's protocol. In brief, the whole eye tissue was rinsed with Phosphatebuffered saline (137 mM NaCl, $2.7 \mathrm{mM} \mathrm{KCl}, 8 \mathrm{mM} \mathrm{Na} \mathrm{HPO}_{4}$, and $2 \mathrm{mM} \mathrm{KH}_{2} \mathrm{PO}_{4}$ ) resuspended in ice cold Mammalian Lysis Buffer (Abcam) and homogenized using Tissuelyser ${ }^{\text {TM }}$ (QIAGEN, Venlo, Netherlands) at a frequency of $30 \mathrm{~Hz}$ for 2 minutes at $4^{\circ} \mathrm{C}$. The homogenate was centrifuged at $10,000 \mathrm{~g}$ for $30 \square$ minutes at $4{ }^{\circ} \mathrm{C}$ and GSH and GSSG levels were measured in the supernatant. To measure GSH and GSSG, a one-step 
fluorometric reaction of samples with respective assay buffer and probes was incubated for $30 \square$ minutes protected from light. At the end of the incubation period, fluorescence intensity was monitored at EX/EM of $490 / 520 \square \mathrm{nm}$ fluorescence on a microplate reader (Spectramax M3, Molecular Devices). The data are presented as the mean and standard error of the mean. Differences were determined using two-tailed Mann-Whitney test (GraphPad Prism version 7.0a for Mac OS X, GraphPad Software, La Jolla California, USA) with p $<0.05$ being considered significant

\section{Protein Extraction and Western Blotting}

Total cell lysates were generated in RIPA buffer (1\% Nonidet P40, 0.5\% sodium deoxycholate, $0.1 \%$ SDS in PBS) by homogenization with a Tissuelyser ${ }^{\text {TM }}$ (QIAGEN, Venlo, Netherlands) at a frequency of $30 \mathrm{~Hz}$ for 2 minutes at $4{ }^{\circ} \mathrm{C}$. Protein concentrations in the homogenate were quantified using the Pierce BCA Protein Assay Kit (Thermo Fisher Scientific) according to the manufacturer's protocol. $10 \mu \mathrm{g}$ of protein was resolved on 4$20 \%$ SDS-PAGE gradient gels (Bio-Rad, Hercules, CA) at $100 \mathrm{~V}$ and then transferred to a $0.2 \mu \mathrm{m}$ nitrocellulose blot (Bio-Rad, Hercules, CA) with the Trans-blot Turbo Transfer System (Bio-Rad, Hercules, CA). The immunoblotting was performed as previously described ([31]) with antibodies against GCLC (Abcam; ab190685) and $\alpha$ A-crystallin (Santa Cruz; sc-28306).

\section{Determining Eye Mass}

To determine the mass of eyes from Gclc WT, Gclc KO, and WT/Cre mice, eyes were enucleated at P21. Following removal of non-ocular tissues by dissection, the eyes were subsequently weighed (Mettler Toledo NewClassic MF). Data are represented as scatter plots. Each point is the average weight of both the left and right eye from one mouse. Differences were determined using ANOVA (GraphPad Prism version 7.0a for Mac OS X, GraphPad Software, La Jolla California, USA), with $\mathrm{p} \leq 0.05$ being considered significant.

\section{Preparing of Eyes for Imaging Mass Spectrometry (IMS)}

P3 and P21 Gclc WT, Gclc HET, or Gclc KO mice were anesthetized and their eyes enucleated and frozen by placing them in a plastic weighing boat floated on liquid nitrogen. The frozen eyes were shipped to Vanderbilt University on dry ice where they were embedded by rapid freezing in $2.6 \%$ carboxymethyl cellulose (Sigma Aldrich, St. Louis, MO, USA). This was accomplished by placing the frozen tissue into an aluminum weight boat (Electron Microscopy Sciences, Hatfield, PA, USA) on dry ice in a polystyrene container and slowly adding carboxymethyl cellulose with a pasture pipette until the eye was coated on all sides. Twelve $\mu \mathrm{m}$ sections were obtained in a sagittal orientation using a cryostat (CM3050S, Leica Biosystems, Buffalo Grove, IL). Triplicate adjacent sections were taken from the central region of the same eye and mounted onto a polylysinecoated Indium-Tin-Oxide (ITO) slide (Delta Technologies, Loveland, CO).

\section{IMS on Prepared Eye Tissue}

Lipid imaging experiments were performed by sublimating the matrices 2,5-dihydroxyacetophenone (DHA) (Sigma Aldrich, St. Louis, MO, USA) 1,5-diaminonaphthalene (DAN) (Sigma Aldrich) onto the samples using an in-house sublimation apparatus [30]. Ten minutes at approximately 56 mTorr at 110 oC for DHA provided a suitable coating for a 10-micron pixel size in positive ion mode. DAN was sublimated for 15 minutes under the same conditions for negative ion mode analysis. Lipid imaging experiments were performed using a rapifleX ${ }^{\mathrm{TM}}$ 
MALDI Tissuetyper ${ }^{\mathrm{TM}}$ (Bruker Daltonics, Billerica, MA) MALDI TOF instrument equipped with a $355 \mathrm{~nm}$ Smartbeam 3D laser operated at $10 \mathrm{kHz}$. Data were acquired in positive and negative ion mode with a $15 \mu \mathrm{m}$ pixel size and 200 shots per pixel over a mass range from 300-1300 Da for negative ion and 200-1300 Da for positive ions.

Protein imaging experiments were performed on tissue sections that were prepared using serial washes for 30 seconds each in 70\% ethanol, $100 \%$ ethanol Carnoy's fluid (60\% ethanol, $30 \%$ chloroform, $10 \%$ acetic acid (2 minutes), $100 \% \mathrm{H}_{2} \mathrm{O}$, and $100 \%$ ethanol. The tissue was air-dried before 2,5-DHA (Sigma Aldrich) matrix (10 $\mathrm{mg} / \mathrm{mL}$ in a solution comprising $7 \mathrm{~mL}$ acetone, $1.5 \mathrm{~mL} \mathrm{H}_{2} \mathrm{O}, 0.4 \mathrm{~mL}$ trifluroacetic acid TFA, and $0.4 \mathrm{~mL}$ acetic acid) was applied by a HTX TM sprayer (HTX Technologies, Carrboro, NC). Four matrix spray passes were made over the tissue with a flow rate of $1.5 \mathrm{~mL} / \mathrm{min}$ at $30^{\circ} \mathrm{C}$ at a track speed of $1300 \mathrm{~cm} / \mathrm{min}$ and a track spacing of $2.5 \mathrm{~mm}$. Data were acquired in positive ion mode using the rapifleX ${ }^{\mathrm{TM}}$ MALDI Tissuetyper ${ }^{\mathrm{TM}}$ with a $20 \mu \mathrm{m}$ pixel size and 100 shots per pixel over a mass range of 1600-2000 Da.

MALDI images were generated using FlexImaging 5.0 (Bruker Daltonics, Billerica, MA) and data were normalized to the total ion current. Relative intensities were adjusted on an individual basis to improve image quality.

\section{Gene Suppression in Zebrafish Embryos}

Splice-blocking morpholinos (sbMOs) targeting either the zebrafish (Danio rerio) gclc exon 1 and exon 5 were designed and synthesized by Gene Tools LLC (Philomath, OR). To determine the optimal sbMO dose, we injected $1 \mathrm{~nL}$ of increasing amounts of each MO $(3 \mathrm{ng} / \mathrm{uL}, 6 \mathrm{ng} / \mathrm{uL}$, and $9 \mathrm{ng} / \mathrm{uL}$ of injection cocktail) into EkxAB embryos (1- to 4-cell stage) harvested from natural mating. To determine MO efficiency, we used TRIzol (Thermo Fisher) to extract total RNA from embryos at 3 days post-fertilization (dpf) according to the manufacturer's instructions. Resulting total RNA was reverse transcribed into cDNA using the Superscript III Reverse Transcriptase kit (Thermo Fisher) and used as template in RT-PCR reactions to amplify regions flanking MO target sites. $10 \mathrm{ng}$ of the RT-PCR product were run on a $2 \%$ agarose gel to determine to the effect of the sbMOs. RT-PCR products were cloned (TOPO-TA; Invitrogen), and the plasmids purified from individual colonies was/were Sanger-sequenced according to standard protocols to identify the precise alteration of the endogenous gclc transcript.

\section{CRISPR/Cas9 Genome Editing in Zebrafish Embryos}

We used CHOPCHOP [32] to identify a guide (g)RNA targeting exon3 of gclc. gRNAs were in vitrotranscribed using the GeneArt precision gRNA synthesis kit (Thermo Fisher) according to the manufacturer's instructions. Zebrafish embryos were obtained from EKxAB embryos harvested from natural mating. One $\mathrm{nL}$ of injection cocktail (100 pg/nl gRNA and $200 \mathrm{pg} / \mathrm{nl}$ Cas9 protein (PNA Bio) in water) was injected into the embryos at the 1-cell stage. To determine targeting efficiency in founder (F0) mutants, we extracted genomic DNA from 2 days post-fertilization (dpf) embryos and PCR amplified the region flanking the gRNA target site. To estimate the percentage of mosaicism of gclc F0 mutants, PCR products were Sanger sequenced according to standard procedures. Cutting efficiency was assessed from sequencing chromatograms using TIDE (Tracking of Indels by DEcomposition) analysis [33].

\section{Phenotypic Analyses in Zebrafish}


To study eye development, larval batches were reared at $28^{\circ} \mathrm{C}$ and imaged live at 3 days-post fertilization (dpf) using the Vertebrate Automated Screening Technology Bioimager (VAST; Union Biometrica) mounted on an AxioScope A1 (Zeiss) microscope using an Axiocam 503 monochromatic camera and Zen Pro 2012 software (Zeiss) as described previously [34]. We obtained lateral brightfield images of whole larvae using the VAST onboard camera and the Axiocam 503 camera. Eye area was automatically measured using FishInspector [35]. The FishInspector Framework provides algorithms and routines for automatic feature-detection from images recorded by the VAST Bioimager Platform; this allowed for unbiased evaluation of statistically-relevant sample sizes. The data are presented using scatter plots. Differences were determined using ANOVA with Tukey correction (GraphPad Prism version 8.0 for Mac OS X, GraphPad Software, La Jolla California, USA), with p < 0.05 being considered significant.

\section{Results}

\section{Lens-specific Gclc deletion reduces whole eye GSH content.}

We successfully generated three genotypes, Gclc WT, Gclc HET and Gclc KO, by crossing Gclc ${ }^{f / f}$ mice with $\mathrm{Le}-\mathrm{Cr} \mathrm{Tg}^{\mathrm{Tg}-}$ (Fig. 1A). As expected from previous results [24], the conditional deletion of Gclc did not cause embryonic lethality, i.e., all three genotypes were born in the expected Mendelian frequency (data not shown). Immunohistochemical staining of GCLC in the eyes of E14.5 mice confirmed that GCLC is abundant in the developing eye, and GCLC expression is absent from the surface ectoderm-derived tissues of Gclc KO mice (Fig. 1B). Western blot analysis at P0 confirmed the absence of GCLC expression in the lenses of Gclc KO mice. Interestingly, Gclc KO retina showed decreased GCLC expression (Fig. 1B and 1C). The lenses of Gclc Het mice have reduced GCLC expression compared with those of Gclc WT mice (Fig. 1C). GCLC binds to GCLM to form the GCL holoenzyme, which catalyzes the rate-limiting step in GSH synthesis. As expected, whole eye levels of GSH and GSSG were markedly reduced in Gclc KO mice (Fig. 1D). The GSH/GSSG ratio in whole eye tissue was not different between Gclc KO and Gclc WT mice (Fig. 1D).

\section{Suppression of Gclc impairs normal eye development.}

To characterize the morphological changes in the eyes of Gclc $\mathrm{KO}$ mice, we conducted gross morphological and histological analysis of eyes at various embryonic and postnatal stages of development (Fig. 2). Gclc WT eyes do not show any ocular abnormalities at any of the ages examined (Figs. 2A, D, G, J). In contrast, Gclc KO mice present age-dependent pathological changes in the lens, cornea, iris, and retina (Figs. 2C, F, I, L). At E14.5, Gclc KO mouse eyes have developed all ocular tissues; however, there is a reduction in the size of the anterior chamber (Fig. 2C, arrow) and increased cellular layers in the retina inner nuclear layer (Fig. 2C, box). By the day of birth (P1), the eyes of Gclc KO mice are drastically altered, with lenses that have severe fiber cell vacuolation (Fig. 2F, arrow), hyperplasia of the iris (Fig. 2F, box), and cellular hyperproliferation of the retina in the ganglion cell, inner plexiform and inner nuclear layers (Fig. 2F, star). The Gclc KO eye abnormalities worsen with age such that by P20, the iris has formed a thick layer that remains attached to the cornea (Fig. 2I, arrow), the lens is smaller in size and vacuolated (Fig. 2I, star), the corneas have thinner epithelial cell layers with hyperproliferation and poor differentiation (Fig. 2I, box), and retinal cellular hyperproliferation is occurring in the outer nuclear, inner nuclear, inner plexiform, and ganglion cell layers (Fig. 2I, circle). By P50, these changes are becoming more profound in Gclc KO mice (Fig. 2L). Interestingly, Gclc KO mice display bilateral microphthalmia whereas Gclc HET mice display only unilateral microphthalmia (note that the unilateral microphthalmia in Gclc HET mice is unbiased towards the right or left eye). In the microphthalmia eye only, Gclc HET mice display identical developmental abnormalities (Fig. 2B, E, H, K) as the Gclc KO mice. 


\section{The microphthalmia phenotype in Le-Cre hemizygous mice is exacerbated by Gclc deletion.}

Our results indicate that Gclc appears to be required for normal eye and lens development. However, Le-Cre transgenic mice can display a microphthalmia phenotype, regardless of floxed alleles [36-39]. Therefore, the extent to which hemizygosity for the Le-Cre causes microphthalmia in our B6/FVB mixed background mice was determined (Fig. 3.) To do so, we bred Gclc ${ }^{f / f} / L e-C r e^{-/-}\left(G c l c\right.$ WT) and Gclc ${ }^{\text {wt/f }} / L e-C r e^{T g /-}$ (Gclc HET) mice (Fig. 1A) for at least three generations to produce Gclc $c^{w t / w t} / \mathrm{Le}-\mathrm{Cr} e^{T g /}$ (WT/Cre) mice, thus isolating the Le-Cre transgene on our B6/FVB mixed background (Fig. 3A). The combined eye weights of Wt/Cre mice and Gclc $\mathrm{KO}$ mice were less $(\mathrm{P}<0.05)$ than those in Gclc WT mice and the weights in Gclc $K O$ mice were less $(\mathrm{P}<0.05)$ than those in WT/Cre mice (Fig. 3B).

\section{Gclc deletion induces molecular changes within the developing eye.}

Immunohistochemical analysis of the eyes of E14.5 day old mice revealed molecular changes in Gclc KO eyes (Fig. 4). Cellular expression of Ki67 (a proliferation marker) was high throughout both the retina and lens epithelium of Gclc WT mice (Fig. 4A). Less staining occurred in the retina and lens epithelium (from the germinative zone to the equator) of Gclc KO mice (Fig. 4E). Apoptotic cells (as revealed by TUNEL staining) were observed within the germinative zone and differentiating secondary fiber cells of Gclc KO eyes (Fig. 4F). No differences in ocular $\beta$-catenin expression were observed between Gclc KO and Gclc WT mice (Fig. 4C \& G). Lenticular Pax6 expression and distribution in Gclc KO eyes (Fig. 4H) appeared to be comparable to that in Gclc WT eyes (Fig. 4D). It should be noted, however, that retinal Pax6 expression was greatly reduced in Gclc $\mathrm{KO}$ eyes (Fig. 4H) relative to Gclc WT eyes (Fig. 4D).

\section{IMS analysis of the whole eye}

Matrix-assisted laser desorption/ionization imaging mass spectrometry (IMS) was employed, in a proof-ofconcept manner, to demonstrate that molecular changes in lipids and proteins can be identified within the eye of a microphthalmia mouse model (Fig. 5). Hematoxylin \& eosin staining of the eyes prepared for IMS (Fig. 5A) displayed the same malformations noted previously (Fig. 2), i.e., Gclc KO eyes have smaller lenses that were vacuolated, exhibited cellular hyperproliferation of the retina, and a decrease in the size of the anterior chamber (Fig. 5A). Analysis of eyes from mice age P21 for changes in protein relative abundance (based on signal intensity) and spatial distribution revealed differences between Gclc $\mathrm{KO}$ and Gclc HET eyes relative to Gclc WT eyes (Fig. 5B). Specifically, thymosin $\beta 4$ (at m/z 4967) signal intensity was reduced in both Gclc KO and HET corneal tissues in a manner that appeared to be Gclc gene dose-dependent, i.e., thymosin $\beta 4$ was reduced to a greater extent in Gclc KO eyes than Gclc HET eyes. (Fig. 5B). Cytochrome C oxidase 7C (at m/z 5449) and histone H2B (at $\mathrm{m} / \mathrm{z} 1378$ ) intensities and distribution were in Gclc KO and Gclc HET mice were similar to Gclc WT mice (Fig. 5B); these proteins clearly illustrate the retinal malformations in Gclc KO eyes.

Analyses of the signal intensity of lipids within eyes of P3 day old mice revealed many differences between Gclc KO and Gclc WT eyes (Figs. 5C,D). Representative images taken in positive ion mode showed a decrease in the signal intensity of an unidentified lipid at m/z 256.8 and an increase in the signal intensity of an unidentified lipid at $\mathrm{m} / \mathrm{z} 525.3$ in the cornea of Gclc $\mathrm{KO}$ mice (Fig. 5C). A decrease in the distribution of putatively-identified phosphatidycholine (PC) $(36: 3)(\mathrm{m} / \mathrm{z}$ at 806.5$)$ in the retina of Gclc KO mice was also observed (Fig. 5C). Analysis of the eyes in negative ion mode identified changes in several lipid species (Fig. 5D). Putatively-identified phosphatidylethanolamine (PE) (40:6) (m/z at 790.5) had decreased signal intensity in the retinas of Gclc KO and Gclc HET mice when compared with Gclc WT mice, however, Gclc KO mice have increased signal intensity when compared with Gclc HET mice. Conversely, the signal intensities for two putatively-identified phosphatidylinositol (PI) lipids (at m/z 863.6 and 885.6) increased in the cornea and retina 
of mice in which Gclc was deleted. Lastly, the signal intensity for putatively-identified NeuAc $\alpha 2-3 \mathrm{Gal} \beta$-Cer was increased in the retinas of Gclc KO mice relative to Gclc WT and Gclc HET mice (Fig. 5D).

\section{A conserved role for $g c l c$ in eye development}

To further investigate the role of GSH in the developing eye, we developed gclc mutant knock-down (KD) and knock out (KO) zebrafish by using splice-blocking morpholinos (KD) or CRISPR/Cas9 genome editing (KO) in zebrafish embryos. Splice-blocking morpholinos (sbMOs) were developed that targeted either zebrafish gclc exon 1 (sb1) or zebrafish exon 5 (sb2) (Suppl. Fig. 1A). Administration of sb1 (3ng was determined to be the optimal dose) caused exon skipping and administration of sb2 (9ng was determined to be the optimal dose) caused intron retention $(219 \mathrm{bp}$ ); these caused $>90 \%$ and $>60 \%$ reductions in $\mathrm{gclc}$ expression, respectively (Suppl. Fig. 1B). Administration of $\mathrm{sb1}$ and $\mathrm{sb} 2$ resulted in a reduction in eye area by $26.5 \%$ and $10 \%$, respectively (Fig. 6A) Representative brightfield images of gclc knock-down fish (3.5 days post fertilization (dpf)) after treatment with either sb1 or sb2 show the reduced eye area (Fig. 6A'). To genetically ablate gclc, CRISPR/Cas9 was utilized with a guide (g)RNA targeting gclc exon 3 (sgRNA1) (Suppl. Fig. 1A). Analyzing the resulting F0 gclc mutants, which are $\approx 30 \%$ mosaic mutants as indicated by CRISPR efficiency (Suppl. Fig. 1C), revealed that the eye area of $3.5 \mathrm{dpf}$ gclc $\mathrm{KO}$ zebrafish is smaller than that of control zebrafish (Fig. 6B). Representative brightfield images of F0 gclc KO fish demonstrates the reduced eye size (Fig. 6B').

\section{Discussion}

In this study, we describe the creation and initial characterization of a conditional Gclc knock-out mouse model involving the use of the Le-Cre transgene (which results in gene deletion being limited to only the lens, cornea, conjunctiva, and eyelid). Additionally, we have described the creation of three gclc-depleted zebrafish models. GCLC is the rate limiting enzyme in glutathione (GSH) biosynthesis. As such, we expected Gclc-depleted animals would have reduced GSH abundance [24-26]. This expectation was confirmed in our Gclc KO mice. Thus, we demonstrate the creation of novel mouse and zebrafish models for the study of the impact of GSH depletion on eye development. Our results indicate that GSH is still present within the developing eye, but at a reduced amount. A failure to see a complete loss of GSH content in the lens was also observed in the LEGSKO mouse model [26]. Further, a large amount of GSH is produced in other ocular tissues besides the lens [40]. In summary, we have successfully deleted Gclc, GCLC protein expression is lost in surface ectoderm-derived tissues, and subsequently whole eye GSH content is reduced.

The microphthalmia phenotype occurring in our Gclc KO mice is starkly different from the cataract phenotype observed in another Gclc knock-out mouse model, the LEGSKO mouse model [26]. LEGSKO mice display reduced lenticular GSH content, elevated ROS, and cataract formation beginning as early as 4 months, but do not have microphthalmia. Analysis of the LEGSKO lens transcriptome revealed that GSH deficiency induces expression of detoxifying genes and activation of epithelial-mesenchymal transition (EMT) signaling [41]. Proteomic analysis of LEGSKO lenses further confirmed the activation of EMT signaling, changes in stress response proteins, and revealed a loss of lens specific markers [42]. The differences in phenotypes between these two mouse models may be explained by the fact that LEGSKO mice and Gclc KO mice have Gclc deletion at different developmental times, however, it is important to note that we did not analyze Gclc KO mice for cataract development at 4 months old. The LEGSKO mouse model relies on the MLR10 Cre transgene to drive gene deletion in lens-specific epithelial and fiber cells at approximately E10.5 [26, 43]. In contrast, our Gclc KO mouse model employs the Le-Cre transgene to drive gene deletion in surface ectoderm-derived tissues 
at approximately E9 [28]. Hence, it is conceivable that the timing of Gclc deletion, and subsequent resultant GSH depletion, may be vitally important for disrupting normal eye development.

Microphthalmia can be caused by developmental defects in both the anterior and posterior segments (total microphthalmia), or by defects in either the anterior or posterior segments (partial microphthalmia) [5]. Gclc KO mice display total microphthalmia, manifesting as severe developmental defects in both the anterior and posterior segments. The deformities observed in the posterior segment were unexpected because Gclc deletion was limited to only surface ectoderm-derived tissues. Mouse models of impaired lens development can display retinal malformations (i.e., infolding and expansion) [28, 45, 46]. Therefore, it is possible that the loss of $G c l c$ from surface ectoderm-derived tissues is disrupting the developmental crosstalk between the lens and the retina.

Gclc KO eyes displayed an age-dependent phenotype that is observable from as early as E14.5 with a decreased anterior chamber size and retinal cellular layer expansion. By the time of weaning (P21), Gclc KO eyes have small vacuolated lens, severe retinal cellular layer expansion and in folding, hypoplasia of the iris, and corneal epithelial cell layers that are hyperproliferated and poorly differentiated. However, mice that have at least one Le-Cre transgene allele may develop microphthalmia regardless of any floxed alleles [36-39]. For example, mice homozygous for the Le-Cre transgene display high penetrance microphthalmia in a strain-dependent manner [38], whereas mice hemizygous for Le-Cre transgene display much lower penetrance in a similarly strain-dependent manner [36, 37]. FVB/N inbred mice served as the original background for the Le-Cre transgene [28] and Le-Cre hemizygous transgenic mice maintained on this background exhibit normal eye development. In contrast, a microphthalmia phenotype appears in Le-Cre hemizygous transgenic mice as the genetic contribution of $\mathrm{FVB} / \mathrm{N}$ is reduced [37, 38]. Given that the Le-Cre transgene utilizes the Pax PO promoter, others have postulated that it may deplete cofactors required for endogenous Pax6 expression [37]. However, a recent study was unable to confirm this hypothesis [36]. Instead, it was found that the microphthalmia phenotype (in Le-Cre homozygous mice) was caused by changes in the expression of genes involved in the negative regulation of cell proliferation and cell growth, and positive regulation of apoptosis pathways. Since these two studies used mice of different strains, the disagreement between them may be explained by strain-dependent differences in Pax6 expression [48] and/or susceptibility to eye development phenotypes when exposed to changes in Pax6 expression or PAX6 levels [48, 49]. Regardless of its cause, it is critical that any study utilizing the Le-Cre transgene control for the effect of the Le-Cre alone. Isolating the LeCre transgene on the mixed B6/FVB background (as used throughout this study) confirmed that the Le-Cre transgene alone causes microphthalmia (Fig. 3B). The present study demonstrates that Gclc deletion during early eye development exacerbates the microphthalmia phenotype of the Le-Cre transgene alone. Our additional observations that Gclc HET mice display unilateral microphthalmia and $g c l c$ deletion in zebrafish causes microphthalmia underscore an important role for Gclc expression in eye development.

Preliminary molecular investigations in Gclc KO eyes revealed a decrease in a cell proliferation marker and increased apoptosis in the developing lens. Cells must proliferate and go through apoptosis for proper organogenesis [50]. Many studies indicate that lens development requires proper cellular proliferation [51-53] and apoptosis [51, 54]. The lens epithelium produces precursor lens fiber cells [55], therefore, a decrease in proliferation of lens epithelial cells will result in a reduction of the lens fiber mass, as observed in Gclc KO mice. Further contributing to the decreased lens fiber mass was an increase in apoptosis in lens epithelial cells and secondary fiber cells. Other studies have shown that changes in PAX6 [56] and $\beta$-catenin [57] expression may cause a loss of lens epithelium proliferation and increased cell death. However, we did not observe changes in the expression of either of these two proteins. A change in the translation of $\alpha$ A-crystallin splice variants in the lenses of Gclc KO mice at P0 was observed. The significance of this within the context of microphthalmia, and the cause of increased cell death and decreased proliferation all remain to be elucidated.

Although the overall phenotype observed in Gclc KO mice differ from those of the LEGSKO mouse model [26, $41,42]$, they are similar to other animal models of oxidative stress. Hydrogen peroxide treatment of lenses obtained from adult glutathione peroxidase (GSHPx) knock-out mice display vacuolated lens epithelial cells in the central region, and degenerated nuclei, organelle loss, and elevated DNA strand breaks in the equatorial 
region of the lens epithelial cells [58]. Similarly, genetic suppression of glutaredoxin 2 (GLRX2) expression (i.e., knock-out) increased the sensitivity of ex vivo mouse lens epithelial cell to oxidative stress and reduced their viability as a result of increased glutathionylation [59]. It is possible that such a process may be occurring within the Gclc KO mice to produce the observed microphthalmia phenotype.

Imaging mass spectrometry (IMS) allows the spatial characterization of thousands of proteins and other small molecules in a tissue in situ during a single experiment [60] (for review, see [61]). This technique has been utilized in investigations of the cornea [62], retinal lipids [63, 64], and lens GSH [65, 66], lipids [67-69], UVfilters [70], and proteins [71, 72]. To our knowledge, our study is the first to use IMS in a microphthalmia mouse model. IMS of eye tissue revealed changes in the distribution and intensities of thymosin $\beta 4$, cytochrome $\mathrm{C}$ oxidase $7 \mathrm{C}$, and histone $\mathrm{H} 2 \mathrm{~B}$. Thymosin $\beta 4$ has a regenerative role (by down-regulating inflammation, inducing tissue regeneration and inhibiting scarring) in the skin, heart, brain and eye [73]. In the eye, thymosin $\beta 4$ promotes corneal wound healing by decreasing inflammation [74], regulating nuclear factor-kappa B (NF$\mathrm{\kappa B})$ in the immune response [75] and regulating matrix metalloproteases [76]. Therefore, the observed loss of thymosin $\beta 4$ expression from the cornea of Gclc $\mathrm{KO}$ mice may render the eyes of these mice with a decreased capacity to respond to exogenous stressors and to heal. Cytochrome $\mathrm{C}$ oxidase 7C (Cox7C), a nuclear encoded subunit of the COX holoenzyme, is highly abundant in mouse retinas [77-79]. It is the terminal enzyme of the electron transport system [80]. Histone H2B is one subunit of the histone $\mathrm{H} 2 \mathrm{~A}-\mathrm{H} 2 \mathrm{~B}$ heterodimer [81], a key histone component [82]. Histone $\mathrm{H} 2 \mathrm{~B}$ is up-regulated in zebrafish retina undergoing regeneration [83]. These proteins are highlighted as markers of the retina, which clearly demonstrate the retinal expansion observed by H\&E staining. IMS analyses also revealed marked changes in the ocular lipid profiles between Gclc KO mice. Phosphatidylinositols (PIs) regulate many processes ranging from; cellular proliferation to death [84]. PIs play a role in lens structural intengrity and photoreceptor cell survival in zebrafish [85].The retinal expansion may explain the increased signal intensity for PI(38:4) within the retina of Gclc KO mice; perhaps they are also important for cell survival within the outer nuclear, inner nuclear, inner plexiform, and ganglion cell layers. It is possible that if Gclc KO mice were observed for long enough that they too would develop a macular dystrophy phenotype. The role of $\mathrm{PI}(36: 1)$ in corneal tissues is unknown, however, it may also regulate cell signaling and could explain the corneal abnormalities observed. While, PIs signal intensities did not differ in the lenses of Gclc KO mice, phosphatidylinositol 3-kinase is necessary for the survival and differentiation of lens fiber cells [86]. Phosphatidylcholines (PCs) are important for rod function, longevity, and function; a shift in their composition is associated with a mouse model for Stargardt-like macular dystrophy [87]. Gangliosides are also known to be abundant throughout the retina, and change as the retina matures [88]. The increased NeuAc $\alpha 2-$ $3 \mathrm{Gal} \beta$-Cer signal intensity in Gclc KO retinas may result from the retinal layer expansion. It is hoped that with continued studies of lipids throughout eye development by IMS, that the role for Gclc in regulating ocular lipid composition will be elucidated.

Gclc is highly expressed in the zebrafish eye throughout embryonic development [29], and is involved in the zebrafish antioxidant response to xenobiotic exposure [89-91]. Herein, we present a novel role for gclc in regulating zebrafish eye development; a reduction in $g c l c$ expression causes reduced eye area (microphthalmia). Thus, demonstrating a role for Gclc in regulating eye development that is conserved throughout the chordate family. The fact that $g c l c \mathrm{KD}$ fish display a more severe phenotype than $g c l c \mathrm{KO}$ fish can be explained by the mosaicism of gclc KO fish and the genetic compensation mechanisms activated by gene KO systems but not gene KD systems [92, 93]. Our novel gclc deficient zebrafish models may be used to provide important insights into human eye development, similar to the recently-described dnajbla zebrafish model. In patients with Peter's Anomaly, DNAJB1 transcripts were shown to be a target of FOXE3. Zebrafish deficient in dnajbla (the zebrafish DNAJB1 ortholog) were used to confirm the role of DNAJB1 in this condition [94]. It is anticipated that $g c l c$-depleted zebrafish will similarly provide unique insights into human lens development and thereby provide benefits for human health.

The present study provides compelling evidence that the loss of Gclc results in severe microphthalmia which is associated with profound molecular changes. In so doing, it represents the first description of a novel role for 
Gclc in eye development. Thus, Gclc KO mice and gclc-depleted zebrafish are valuable new models for studying microphthalmia. Investigations in these animal models, utilizing powerful next-generation technologies (IMS, RNA-seq, and redox proteomics), are ongoing. It is expected that these studies will accelerate the development of new strategies for the prevention of and treatment of microphthalmia.

\section{References}

1. Hornby S, Gilbert CE, Rahi JS, Sil A, Xiao Y, Dandona L, et al. Regional variation in blindness in children due to microphthalmos, anophthalmos and coloboma. Opthalmic Epidemiology. 2000;7:127-38.

2. Parker SE, Mai CT, Canfield MA, Rickard R, Wang Y, Meyer RE, et al. Updated national birth prevalence estimates for selected birth defects in the United States, 2004-2006. Birth Defects Research Part A: Clinical and Molecular Teratology. 2010;88:1008-16.

3. Plaisancié J, Ceroni F, Holt R, Zazo Seco C, Calvas P, Chassaing N, et al. Genetics of anophthalmia and microphthalmia. Part 1: Non-syndromic anophthalmia/microphthalmia. Human Genetics. 2019.

4. International Clearinghouse for Birth Defects Monitoring Systems. Annual Report 20142014; Rome.

5. Warburg M. Classification of microphthalmos and coloboma. Journal of Medical genetics. 1993;30:664-

9.

6. Cvekl A, Ashery-Padan R. The cellular and molecular mechanisms of vertebrate lens development. Development. 2014;141:4432-47.

7. Williamson KA, FitzPatrick DR. The genetic architecture of microphthalmia, anophthalmia and coloboma. European Journal of Medical Genetics. 2014;57:369-80.

8. Smithells RW. Defects and disabilities of thalidomide children. British Medical Journal. 1973;1:269-72.

9. Wertelecki W, Koerblein A, levtushok B, Zymak-Zakutnia N, Komov O, Kuznietsov I, et al. Elevated congenital anomaly rates and incorporated cesium-137 in the Polissia region of Ukraine. Birth Defects Research Part A: Clinical and Molecular Teratology. 2016;106(3):194-200.

10. Craenen K, Verslegers M, Buset J, Baatout S, Moons L, Benotmane MA. A detailed characterization of congenital defects and mortality following moderate X-ray doses during neurulation. Birth Defects Research. 2018;110(6):467-82.

11. Gilbert R. "Clusters" of anophthalmia in Britain. BMJ (Clinical research ed). 1993;307:340-1.

12. Vermeij-Keers C. Primary congenital aphakia and the rubella syndrome. Teratology. 1975;11:257-65.

13. Smets KJ, Barlow T, Vanhaesebrouck P. Maternal vitamin A deficiency and neonatal microphthalmia: complications of biliopancreatic diversion? European Journal of Pediatrics. 2006;165(7):502-4.

14. Cools M, Duval ELIM, Jespers A. Adverse neonatal outcome after maternal biliopancreatic diversion operation: report of nine cases. European Journal of Pediatrics. 2006;165(3):199-202.

15. Llorente-González S, Peralta-Calvo J, Abelairas-Gómez JM. Congenital anophthalmia and microphthalmia: Epidemiology and orbitofacial rehabilitation. Clinical Ophthalmology. 2011;5:1759-65.

16. Wang X, Gregory-evans K, Wasan KM, Sivak O, Shan X, Gregory-evans CY. Efficacy of postnatal in vivo nonsense suppression therapy in a Pax6 mouse model of aniridia. Molecular Therapy: Nucleic Acid. 2017;7:417-28.

17. GIBLIN FJ. Glutathione: A vital lens antioxidant. Journal of Ocular Pharmacology and Therapeutics. 2000;16:121-35.

18. Giblin F, Chakrapani B, VN R. Glutathione and lens epithelial function. Investigative Ophthalmology and Visual Science. 1976;15:381-93.

19. Dalton TP, Chen Y, Schneider SN, Nebert DW, Shertzer HG. Genetically altered mice to evaluate glutathione homeostasis in health and disease. Free Radical Biology and Medicine. 2004;37:1511-26.

20. Truscott RJW, Augusteyn RC. The state of sulphydryl groups in normal and cataractous human lenses. Experimental Eye Research. 1977;25:139-48. 
21. Sweeney MHJ, Truscott RJW. An Impediment to Glutathione Diffusion in Older Normal Human Lenses: a Possible Precondition for Nuclear Cataract. Experimental Eye Research. 1998;67:587-95.

22. Meister A, Anderson ME. Glutathione. Annual Review of Biochemistry. 1983;52:711-60.

23. Yang $Y$, Dieter MZ, Chen Y, Shertzer HG, Nebert DW, Dalton TP. Initial characterization of the glutamate-cysteine ligase modifier subunit $\mathrm{Gclm}(-/-)$ knockout mouse. Journal of Biological Chemistry. 2002;277:49446-52.

24. Chen Y, Yang Y, Miller ML, Shen D, Shertzer HG, Stringer KF, et al. Hepatocyte-specific Gclc deletion leads to rapid onset of steatosis with mitochondrial injury and liver failure. Hepatology. 2007;45:1118-28.

25. Feng W, Rosca M, Fan Y, Hu Y, Feng P, Lee HG, et al. Gclc deficiency in mouse CNS causes mitochondrial damage and neurodegeneration. Hum Mol Genet. 2017;26:1376-90.

26. Fan X, Liu X, Hao S, Wang B, Robinson ML, Monnier VM. The LEGSKO Mouse: A mouse model of agerelated nuclear cataract based on genetic suppression of lens glutathione synthesis. PLoS One. 2012;7:1-9.

27. Feng $W$, Rosca $M$, Fan $Y$, Hu $Y$, Feng $P$, Lee $H-G$, et al. Gclc deficiency in mouse CNS causes mitochondrial damage and neurodegeneration. Hum Mol Genet. 2017;26(7):1376-90.

28. Ashery-Padan R, Marquardt T, Zhou X, Gruss P. Pax6 activity in the lens primordium is required for lens formation and for correct placement of a single retina in the eye. Genes and Development. 2000;14:2701-11.

29. Timme-Laragy AR, Goldstone JV, Imhoff BR, Stegeman JJ, Hahn ME, Hansen JM. Glutathione redox dynamics and expression of glutathione-related genes in the developing embryo. Free Radic Biol Med. 2013;65:89-101.

30. Thisse B, Pflumio S, Fürthauer M, Loppin B, Heyer V, Degrave A, et al. Expression of the zebrafish genome during embryogenesis (NIH R01 RR15402). 2001.

31. Zullo AJ, Lee S. Mycobacterial induction of autophagy varies by species and occurs independently of mammalian target of rapamycin inhibition. J Biol Chem. 2012;287(16):12668-78.

32. Labun K, Montague TG, Gagnon JA, Thyme SB, Valen E. CHOPCHOP v2: a web tool for the next generation of CRISPR genome engineering. Nucleic Acids Res. 2016;44(W1):W272-W6.

33. Brinkman EK, Chen T, Amendola M, van Steensel B. Easy quantitative assessment of genome editing by sequence trace decomposition. Nucleic Acids Res. 2014;42(22):e168-e.

34. Shaw ND, Brand H, Kupchinsky ZA, Bengani H, Plummer L, Jones TI, et al. SMCHD1 mutations associated with a rare muscular dystrophy can also cause isolated arhinia and Bosma arhinia microphthalmia syndrome. Nat Genet. 2017;49(2):238-48.

35. Teixidó E, Kießling TR, Krupp E, Quevedo C, Muriana A, Scholz S. Automated morphological feature assessment for zebrafish embryo developmental toxicity screens. Toxicol Sci. 2019;167(2):438-49.

36. Lam PT, Padula SL, Hoang TV, Poth JE, Liu L, Liang C, et al. Considerations for the use of Cre recombinase for conditional gene deletion in the mouse lens. Human Genomics. 2019;13:1-17.

37. Dorà NJ, Collinson JM, Hill RE, West JD. Hemizygous Le-Cre transgenic mice have severe eye abnormalities on some genetic backgrounds in the absence of LoxP Sites. PLoS One. 2014;9.

38. Robinson ML, Walton NA, Wallace LM, Yang Y. Homozygosity for the Le-Cre transgene is associated with microphthalmia and cataracts in the absence of other engineered genetic changes. Investigative Ophthalmology and Visual Science. 2005;46:1885.

39. De Maria A, Bassnett S. Birc7: A late fiber gene of the crystalline lens. Investigative Ophthalmology and Visual Science. 2015;56:4823-34.

40. Whitson JA, Sell DR, Goodman MC, Monnier VM, Fan X. Evidence of dual mechanisms of glutathione uptake in the rodent lens: a novel role for vitreous humor in lens glutathione homeostasis. Investigative Ophthalmology and Visual Science. 2016;57:3914-25.

41. Whitson JA, Zhang X, Medvedovic M, Chen J, Wei Z, Vincent M, et al. Transcriptome of the GSHdepleted lens reveals changes in detoxification and EMT signaling genes, transport systems, and lipid homeostasis. Investigative Ophthalmology and Visual Science. 2017;58:2666-84. 
42. Whitson JA, Wilmarth PA, Klimek J, Monnier VM, David L, Fan X. Proteomic analysis of the glutathionedeficient LEGSKO mouse lens reveals activation of EMT signaling, loss of lens specific markers, and changes in stress response proteins. Free Radical Biology and Medicine. 2017;113:84-96.

43. Zhao H, Yang Y, Rizo CM, Overbeek PA, Robinson ML. Insertion of a Pax6 consensus binding site into the A-crystallin promoter acts as a lens epithelial cell enhancer in transgenic mice. Investigative Ophthalmology and Visual Science. 2004;45:1930-9.

44. Feil R, Brocard J, Mascrez B, LeMeur M, Metzger D, Chambon P. Ligand-activated site-specific recombination in mice. Proceedings of the National Academy of Sciences. 1996;93:10887 LP-90.

45. Manuel M, Pratt T, Liu M, Jeffery G, Price DJ. Overexpression of Pax6 results in microphthalmia, retinal dysplasia and defective retinal ganglion cell axon guidance. BMC Developmental Biology. 2008;8:59.

46. Gregory-evans CY, Wang X, Wasan KM, Zhao J, Metcalfe AL, Gregory-evans K. Postnatal manipulation of Pax6 dosage reverses congenital tissue malformation defects. Journal of Clinical Investigation. 2014;124:27.

47. Klimova L, Lachova J, Machon O, Sedlacek R, Kozmik Z. Generation of mRx-Cre transgenic mouse line for efficient conditional gene deletion in early retinal progenitors. PLoS One. 2013;8:e63029-e.

48. Hickmott JW, Gunawardane U, Jensen K, Korecki AJ, Simpson EM. Epistasis between Pax6(Sey) and genetic background reinforces the value of defined hybrid mouse models for therapeutic trials. Gene Therapy. 2018;25(8):524-37.

49. Chanas SA, Collinson JM, Ramaesh T, Dorà N, Kleinjan DA, Hill RE, et al. Effects of elevated Pax6 expression and genetic background on mouse eye development. Investigative Opththalmology \& Visual Science. 2009;50(9):4045-59.

50. Fuchs Y, Steller H. Programmed Cell Death in Animal Development and Disease. Cell. 2011;147(4):742-

58.

51. Baker NE. Cell proliferation, survival, and death in the Drosophila eye. Seminars in Cell \& Developmental Biology. 2001;12(6):499-507.

52. Medina-Martinez O, Brownell I, Amaya-Manzanares F, Hu Q, Behringer RR, Jamrich M. Severe defects in proliferation and differentiation of lens cells in Foxe3 null mice. Mol Cell Biol. 2005;25(20):8854-63.

53. Cavalheiro GR, Matos-Rodrigues GE, Gomes AL, Rodrigues PMG, Martins RAP. c-Myc regulates cell proliferation during lens development. PLoS One. 2014;9(2):e87182-e.

54. Chaffee BR, Hoang TV, Leonard MR, Bruney DG, Wagner BD, Dowd JR, et al. FGFR and PTEN signaling interact during lens development to regulate cell survival. Dev Biol. 2016;410(2):150-63.

55. Cvekl A, Ashery-Padan R. The cellular and molecular mechanisms of vertebrate lens development. Development. 2014;141(23):4432.

56. Shaham O, Smith AN, Robinson ML, Taketo MM, Lang RA, Ashery-Padan R. Pax6 is essential for lens fiber cell differentiation. Development. 2009;136(15):2567-78. Epub 2009/07/01.

57. Cain S, Martinez G, Kokkinos MI, Turner K, Richardson RJ, Abud HE, et al. Differential requirement for $\beta$-catenin in epithelial and fiber cells during lens development. Dev Biol. 2008;321(2):420-33.

58. Reddy VN, Giblin FJ, Lin L-R, Dang L, Unakar NJ, Musch DC, et al. Glutathione Peroxidase-1 Deficiency Leads to Increased Nuclear Light Scattering, Membrane Damage, and Cataract Formation in Gene-Knockout Mice. Investigative Ophthalmology \& Visual Science. 2001;42:3247-55.

59. Wu H, Lin L, Giblin F, Ho Y-S, Lou MF. Glutaredoxin 2 knockout increases sensitivity to oxidative stress in mouse lens epithelial cells. Free Radic Biol Med. 2011;51(11):2108-17.

60. Caprioli RM, Farmer TB, Gile J. Molecular Imaging of Biological Samples:? Localization of peptides and proteins using MALDI-TOF MS. Analytical Chemistry. 1997;69:4751-60.

61. Buchberger AR, DeLaney K, Johnson J, Li L. Mass Spectrometry Imaging: a review of emerging advancements and future insights. Analytical chemistry. 2018;90:240-65.

62. Chen Y, Jester JV, Anderson DM, Marchitti SA, Schey KL, Thompson DC, et al. Corneal haze phenotype in Aldh3a1-null mice: In vivo confocal microscopy and tissue imaging mass spectrometry. Chemico-Biological Interactions. 2017;276:9-14. 
63. Andersen M, Anderson H, Bailar JC, Boekelheide K, Brent R, Charnley G, et al. Toxicity testing in the 21St century: a vision and a strategy. National Academy of Science. 2007;13:51-138.

64. Ly A, Schöne C, Becker M, Rattke J, Meding S, Aichler $M$, et al. High-resolution MALDI mass spectrometric imaging of lipids in the mammalian retina. Histochemistry and Cell Biology. 2015;143:453-62.

65. Grey AC, Demarais NJ, West BJ, Donaldson PJ. A quantitative map of glutathione in the aging human lens. International Journal of Mass Spectrometry. 2019;437:58-68.

66. Nye-Wood MG, Spraggins JM, Caprioli RM, Schey KL, Donaldson PJ, Grey AC. Spatial distributions of glutathione and its endogenous conjugates in normal bovine lens and a model of lens aging. Experimental Eye Research. 2017;154:70-8.

67. Deeley JM, Hankin JA, Friedrich MG, Murphy RC, Truscott RJW, Mitchell TW, et al. Sphingolipid distribution changes with age in the human lens. Journal of Lipid Research. 2010;51:2753-60.

68. Distribution of Glycerophospholipids in the Adult Human Lens, (2018).

69. Pol J, Faltyskova H, Krasny L, Volný M, Vlacil O, Hajduch M, et al. Age-Related Changes in the Lateral Lipid Distribution in a Human Lens Described by Mass Spectrometry Imaging. European Journal of Mass Spectrometry. 2015;21:297-303.

70. Demarais NJ, Donaldson PJ, Grey AC. Age-related spatial differences of human lens UV filters revealed by negative ion mode MALDI imaging mass spectrometry. Experimental Eye Research. 2019;184:146-51.

71. Grey AC, Schey KL. Age-related changes in the spatial distribution of human lens alpha-crystallin products by MALDI imaging mass spectrometry. Investigative Ophthalmology \& Visual Science. 2009;50:431929. E

72. Stella DR, Floyd KA, Grey AC, Renfrow MB, Schey KL, Barnes S. Tissue localization and solubilities of $\alpha A$ crystallin and its numerous C-terminal truncation products in pre- and postcataractous ICR/f rat lenses. Investigative Ophthalmology \& Visual Science. 2010;51:5153-61.

73. Goldstein AL, Hannappel E, Sosne G, Kleinman HK. Thymosin $\beta 4$ : a multi-functional regenerative peptide. Basic properties and clinical applications. Expert Opinion on Biological Therapy. 2012;12:37-51.

74. Sosne G, Szliter EA, Barrett R, Kernacki KA, Kleinman H, Hazlett LD. Thymosin Beta 4 promotes corneal wound healing and decreases inflammation in vivo following alkali injury. Experimental Eye Research. 2002;74(2):293-9.

75. Qiu P, Wheater MK, Qiu Y, Sosne G. Thymosin beta4 inhibits TNF-alpha-induced NF-kappaB activation, IL-8 expression, and the sensitizing effects by its partners PINCH-1 and ILK. FASEB journal: official publication of the Federation of American Societies for Experimental Biology. 2011;25(6):1815-26.

76. Philp D, Scheremeta B, Sibliss K, Zhou M, Fine EL, Nguyen M, et al. Thymosin $\beta 4$ promotes matrix metalloproteinase expression during wound repair. Journal of Cellular Physiology. 2006;208(1):195-200.

77. Wang A-G, Lee C-M, Wang Y-C, Lin C-H, Fann M-J. Up-regulation of cytochrome oxidase in the retina following optic nerve injury. Experimental Eye Research. 2002;74:651-9.

78. Phillips MJ, Webb-Wood S, Faulkner AE, Jabbar SB, Biousse V, Newman NJ, et al. Retinal function and structure in ant1-deficient mice. Investigative Ophthalmology \& Visual Science. 2010;51:6744-52.

79. Begum R, Powner MB, Hudson N, Hogg C, Jeffery G. Treatment with $670 \mathrm{~nm}$ light up regulates cytochrome $\mathrm{C}$ oxidase expression and reduces inflammation in an age-related macular degeneration model. PLoS One. 2013;8:e57828-e.

80. Little AG, Lau G, Mathers KE, Leary SC, Moyes CD. Comparative biochemistry of cytochrome c oxidase in animals. Comparative Biochemistry and Physiology Part B: Biochemistry and Molecular Biology. 2018;224:170-84.

81. Moriwaki $Y$, Yamane $T$, Ohtomo H, Ikeguchi $M$, Kurita J-I, Sato $M$, et al. Solution structure of the isolated histone H2A-H2B heterodimer. Scientific Reports. 2016;6:24999.

82. Luger K. Structure and dynamic behavior of nucleosomes. Current Opinion in Genetics \& Development. 2003;13:127-35. 
83. Eastlake K, Heywood WE, Tracey-White D, Aquino E, Bliss E, Vasta GR, et al. Comparison of proteomic profiles in the zebrafish retina during experimental degeneration and regeneration. Scientific Reports. $2017 ; 7: 44601$.

84. Balla T. Phosphoinositides: Tiny lipids with giant impact on cell regulation. Physiological Reviews. 2013;93(3):1019-137.

85. Murphy TR, Vihtelic TS, Ile KE, Watson CT, Willer GB, Gregg RG, et al. Phosphatidylinositol synthase is required for lens structural integrity and photoreceptor cell survival in the zebrafish eye. Experimental Eye Research. 2011;93(4):460-74. Epub 2011/06/23.

86. Weber GF, Menko AS. Phosphatidylinositol 3-Kinase Is necessary for lens fiber cell differentiation and survival. Investigative Ophthalmology \& Visual Science. 2006;47(10):4490-9.

87. Bennett LD, Brush RS, Chan M, Lydic TA, Reese K, Reid GE, et al. Effect of reduced retinal VLC-PUFA on rod and cone photoreceptors. Investigative Ophthalmology \& Visual Science. 2014;55(5):3150-7.

88. Fontaine V, Hicks D, Dreyfus H. Changes in ganglioside composition of photoreceptors during postnatal maturation of the rat retina. Glycobiology. 1998;8(2):183-90.

89. Liu H, Gooneratne R, Huang X, Lai R, Wei J, Wang W. A rapid in vivo zebrafish model to elucidate oxidative stress-mediated PCB126-induced apoptosis and developmental toxicity. Free Radical Biology and Medicine. 2015;84:91-102.

90. Arnold MC, Forte JE, Osterberg JS, Di Giulio RT. Antioxidant Rescue of Selenomethionine-induced teratogenesis in zebrafish embryos. Archives of Environmental Contamination and Toxicology. 2016;70:31120.

91. Liu L, Zhu H, Yan Y, Lv P, Wu W. Toxicity evaluation and biomarker selection with validated reference gene in embryonic zebrafish exposed to mitoxantrone. International Journal of Molecular Sciences. 2018;19:3516.

92. Ma Z, Zhu P, Shi H, Guo L, Zhang Q, Chen Y, et al. PTC-bearing mRNA elicits a genetic compensation response via Upf3a and COMPASS components. Nature. 2019;568(7751):259-63.

93. El-Brolosy MA, Kontarakis Z, Rossi A, Kuenne C, Günther S, Fukuda N, et al. Genetic compensation triggered by mutant mRNA degradation. Nature. 2019;568(7751):193-7.

94. Khan SY, Vasanth S, Kabir F, Gottsch JD, Khan AO, Chaerkady R, et al. FOXE3 contributes to Peters anomaly through transcriptional regulation of an autophagy-associated protein termed DNAJB1. Nature Communications. 2016;7:10953. 


\section{Table}

\begin{tabular}{|l|l|}
\hline Abbreviation & Genotype \\
\hline Gclc WT & Gclc $^{f / f} /$ Le-Cre \\
\hline Gclc HET & Gclc $^{\text {wt/f}} /$ Le-Cre \\
\hline Gclc KO & Gclc $^{\text {T/f }} /$ Le-Cre \\
\hline Gclc WT/Cre & Gclc $^{\text {wt } / w t} /$ Le-Cre \\
\hline
\end{tabular}

Table 1. Definitions of abbreviations used for mouse genotypes. 


\section{Main Figures}

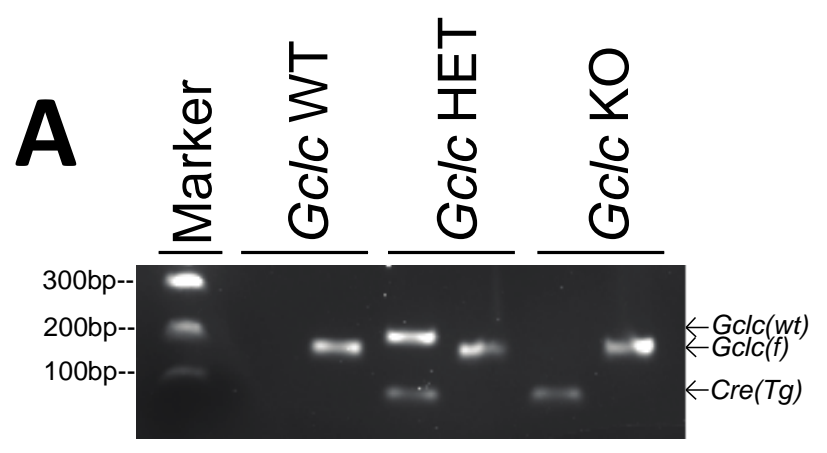

C

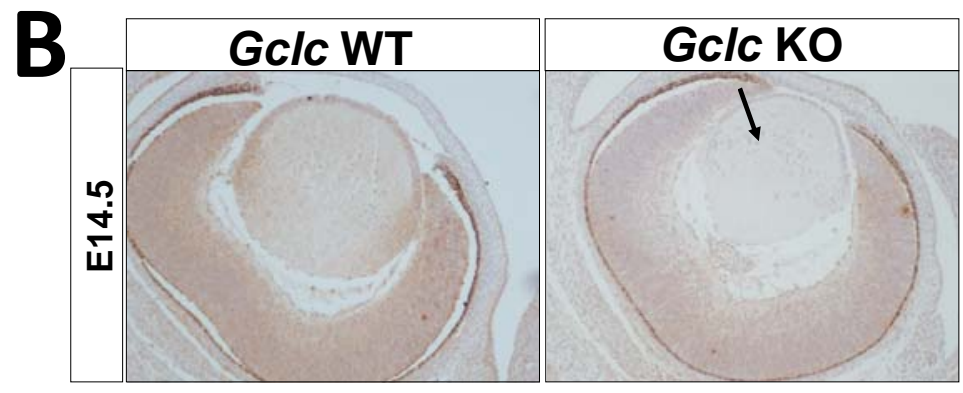

D
GSSG
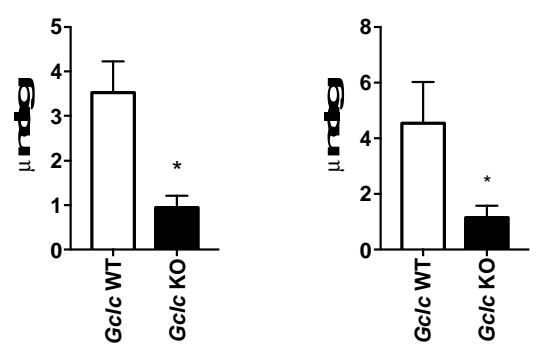

GSH/GSSG

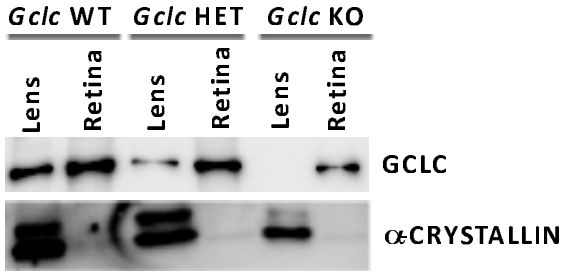

Figure 1. Surface-ectoderm derived tissue-specific deletion of Gclc reduces ocular GSH content

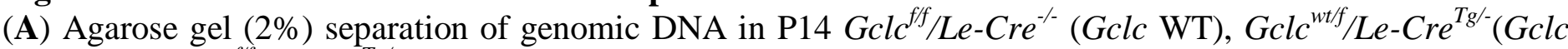
HET), and Gclc ${ }^{f f / L e-C r e e^{T / L}}$ (Gclc KO) mice by PCR amplification of the Gclc wild-type (Gclc(wt)) or Gclc floxed $(G c l c(f))$ alleles, or the Le-Cre transgene $(\mathrm{Cre}(\mathrm{Tg}))$. (B) Immunohistochemical staining of GCLC in E14.5 Gclc WT, Gclc HET and Gclc KO mice (Arrow marks a lens with a loss of GCLC expression; 100x magnification). (C) Western blot analysis of GCLC and $\alpha \mathrm{A}$-crystallin proteins in the lens and retina of P0 Gclc WT, Gclc HET and Gclc KO mice. The upper band observed in the blot stained for $\alpha$ A-crystallin represents an alternatively spliced $\alpha$ A-crystallin variant containing an additional exon. (D) GSH and GSSG levels in the whole eyes of P21 Gclc WT (white bars) and Gclc KO (black bars) mice. The ratio of GSH/GSSG is also shown. Data are presented as the mean and associated standard error of the mean from 3-4 mice. * $\mathrm{P}<0.05$, two-tailed Mann-Whitney test, compared to Gclc WT. 

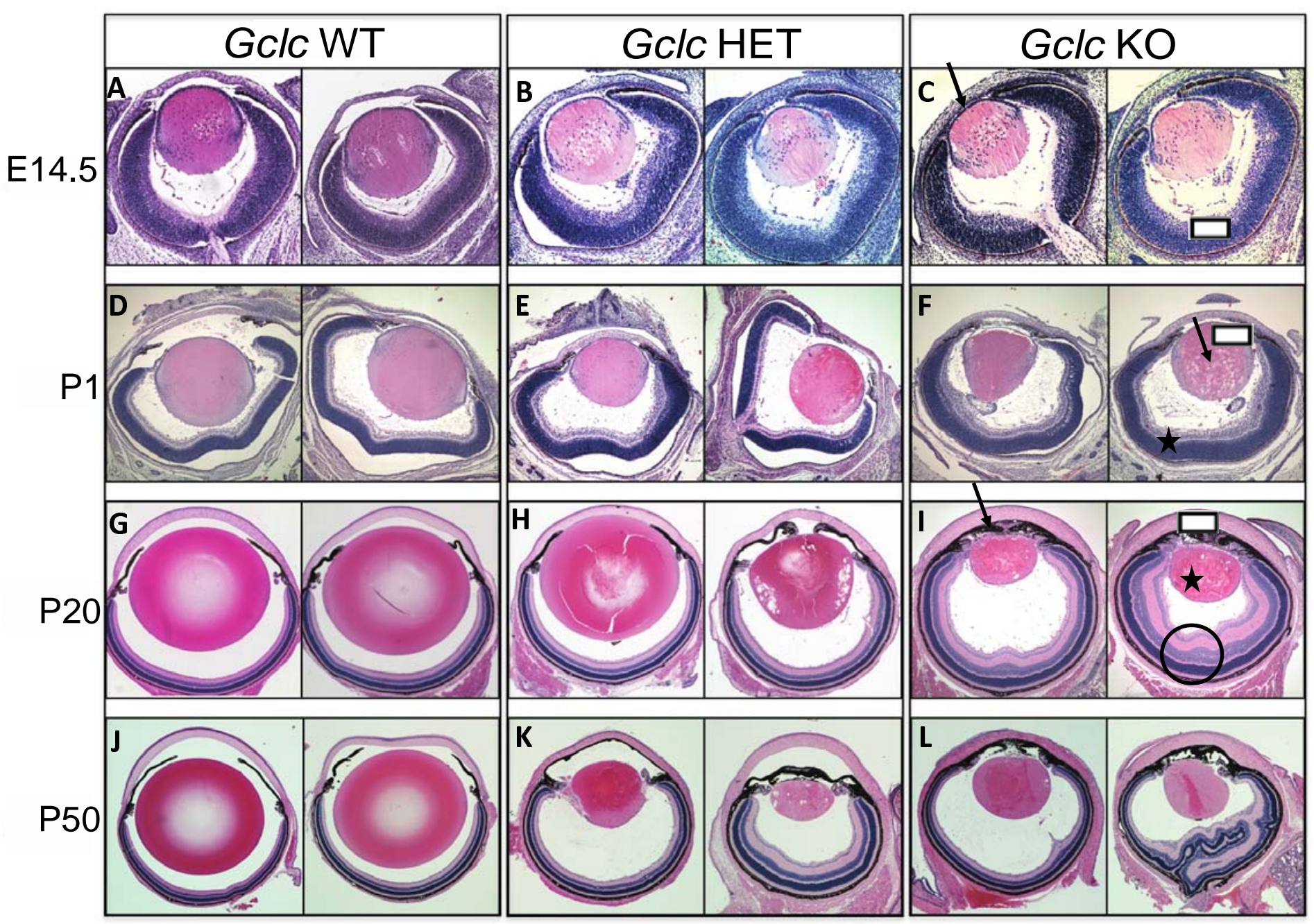

\section{Figure 2. Gclc deletion causes severe ocular malformations}

Representative images of hematoxylin and eosin-stained left (left column) and right (right column) eyes (from the same mouse at each age) from Gclc WT, Gclc HET, and Gclc KO mice at ages E14.5 (A-C), P1 (D-F), P20 (G-I), and P50 (J-L). In C, the arrow marks a reduction in the size of the anterior chamber and the box marks increased cellular layers of the retina inner nuclear layer. In $\mathbf{F}$, the arrow marks vacuolated lens fiber cells, the box marks hyperplasia of the iris, and the star marks hyperproliferation of the retina ganglion cell, inner nuclear, and inner plexiform layers. In I, the star marks a small vacuolated lens, the box marks a cornea with abnormally thin epithelial layers with hyperproliferation and poor differentiation, and the circle marks a retina with severe hyperproliferation in the outer nuclear, inner nuclear, inner plexiform, and ganglion cell layers. Magnification = 100x. 

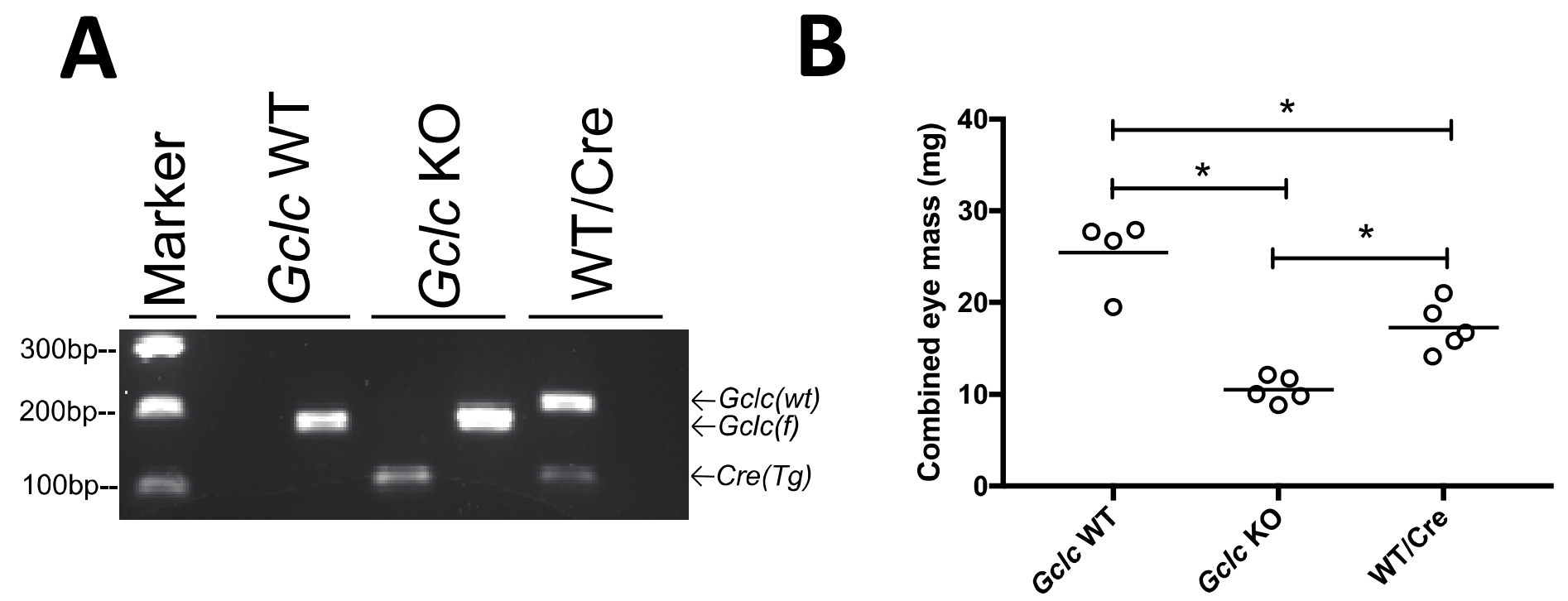

Figure 3. Gclc deletion potentiates the microphthalmia phenotype of the $\mathrm{Le}$-Cre transgene

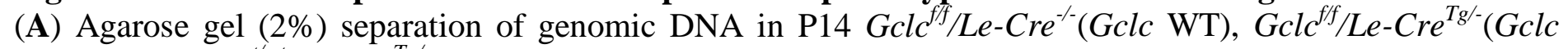
HET), and Gclc $c^{w t / w t} / L e-C r e^{T g /-}$ (WT/Cre) mice by amplification of the Gclc wild-type (Gclc(wt)) or Gclc floxed $(G c l c(f))$ alleles, or the Le-Cre transgene $(C r e(T g))$. Two separate PCR reactions are needed to properly describe each individual animal: one detected the $(w t)$ and $C r e(T g)$ alleles (left lane) and the other the $G c l c(f)$ allele (right lane). (B) Combined (left and right) eye weights from P21 Gclc WT, Gclc KO and WT/Cre mice. Each point represents results from an individual mouse. Horizontal lines indicate the mean. * $\mathrm{P}<0.05$, ANOVA, with Tukey correction, compared to other group indicated.

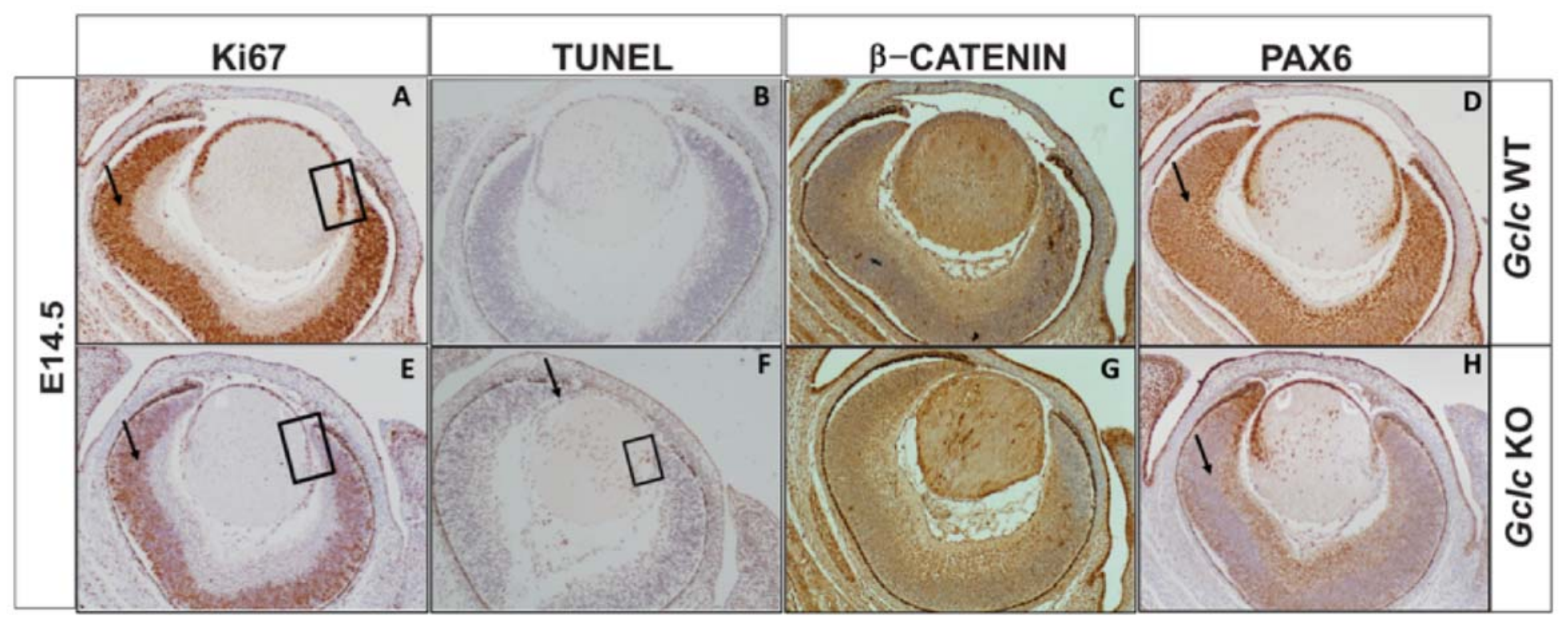


Figure 4. Gclc deletion induces molecular changes during embryonic eye development

Staining of sagittal sections of eyes from E14.5 day old Gclc WT (A-D) and Gclc KO (E-H) mice for Ki67 (A,E), TUNEL (B,F), $\beta$-Catenin $(\mathbf{C}, \mathbf{G})$, or Pax6 (D,H). In $\mathbf{A}$ and $\mathbf{E}$, the brown-staining Ki67-positive (proliferative) cells are identified in the retina (arrow) and in the lens epithelial cells (box). In F, TUNELpositive (apoptotic) cells in the lens epithelium (arrow) and secondary lens fiber cells (box) are shown. In D and $\mathbf{H}$, Pax6-positive cells in the retina are identified by an arrow. (Magnification $=100 \mathrm{x}$ )
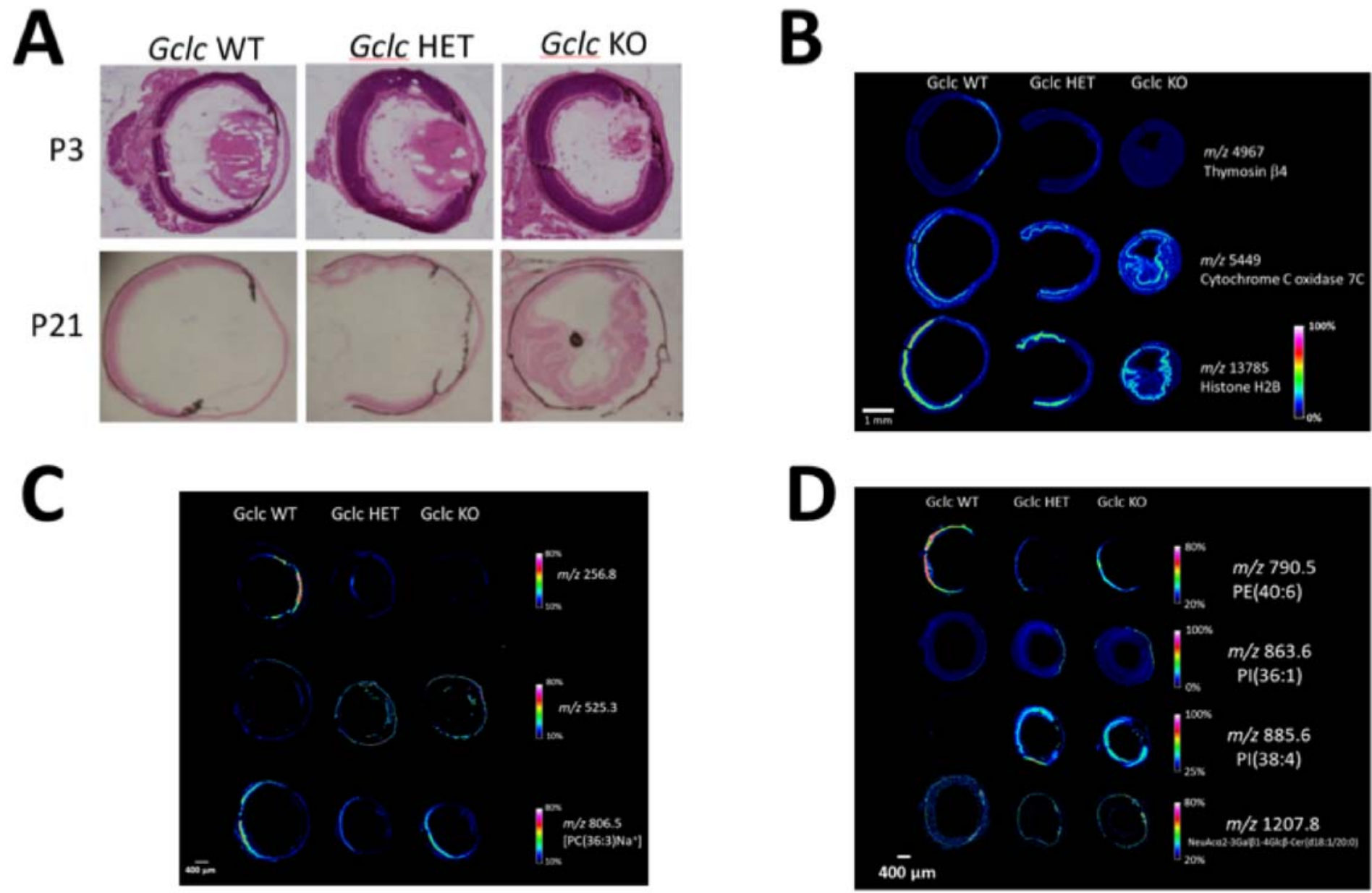

Figure 5. Imaging mass spectrometry (IMS) analysis of the eye

Histology and IMS were conducted in whole eye tissues samples from Gclc WT, Gclc HET, and Gclc KO mice at P3 and P21 days of age. (A) Hematoxylin and eosin-stained tissue sections used for IMS (Magnification = 100x). (B) Representative images indicating the intensity and localization of proteins. (C-D) Representative images indicating the intensity and localization of lipids in positive (C) and negative (D) mode. Individual images represent selected ions $(\mathrm{m} / \mathrm{z})$ and color intensities indicate relative abundances based on the provided color scale. 

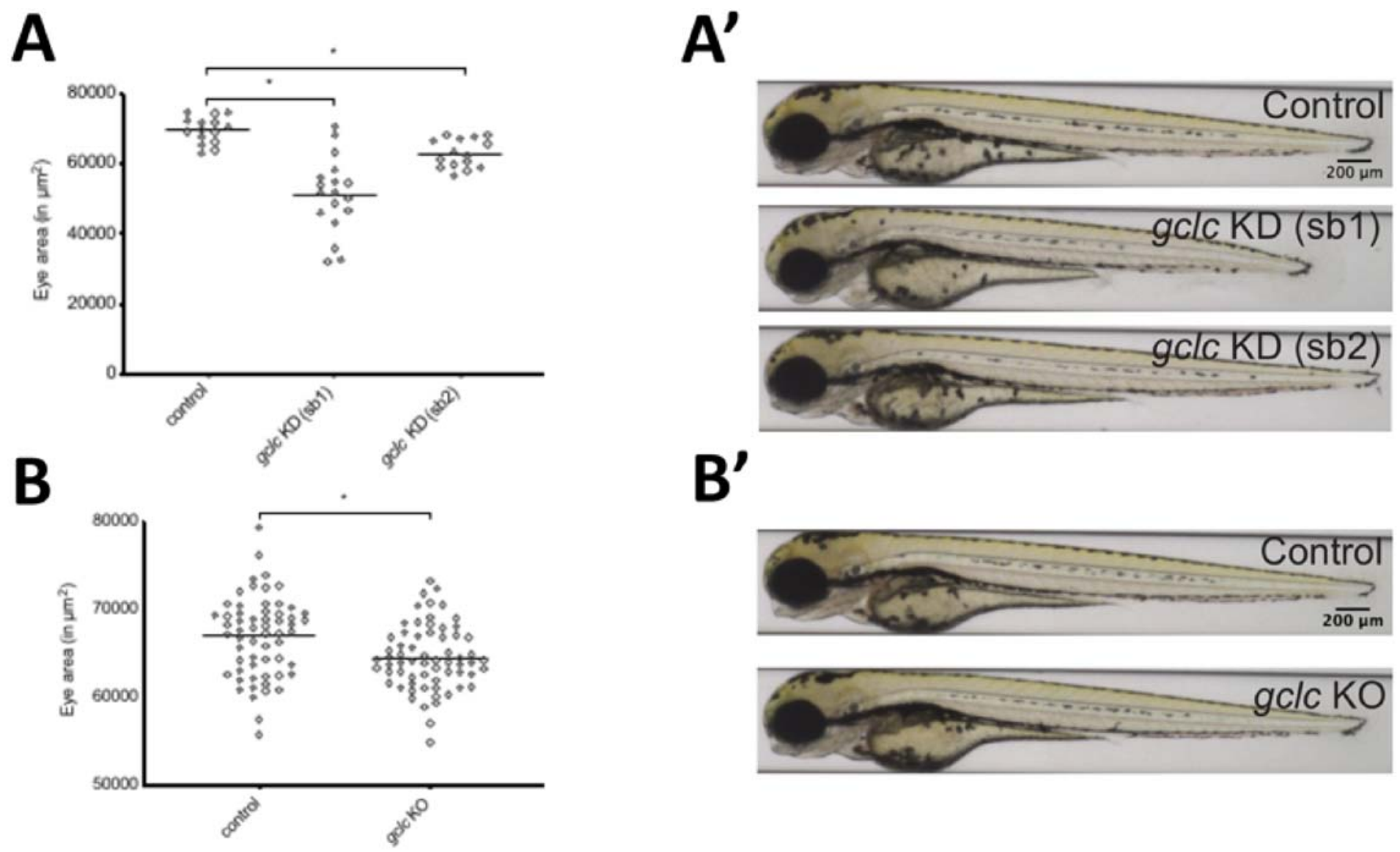

$\mathbf{B}^{\prime}$
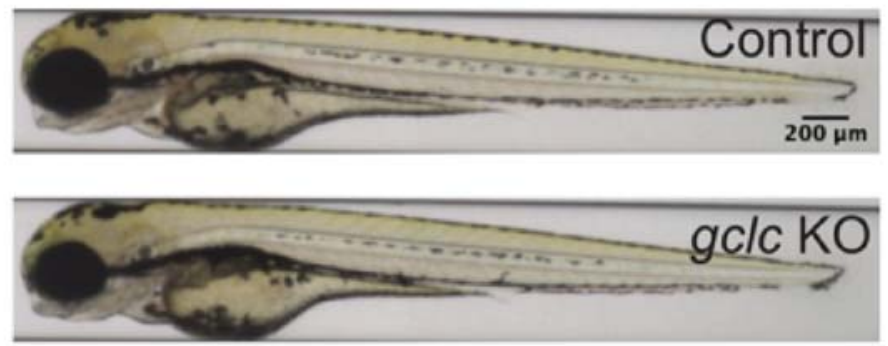

Figure 6. gclc gene suppression induces microphthalmia in zebrafish

(A) Embryonic eye area (in $\mu \mathrm{m}^{2}$ ) at 3.5 days post fertilization (dpf) of control and $g c l c \mathrm{KD}$ fish, i.e., zebrafish in which $g c l c$ had been suppressed by sbMOs ( $g c l c$ KD sb1 and gclc KD sb2 ). (A') Representative brightfield image of the lateral view of $3.5 \mathrm{dpf}$ control and gclc KD fish. (B) Embryonic eye area (in $\mu \mathrm{m}^{2}$ ) of $3.5 \mathrm{dpf}^{\circ}$ control and gclc KO fish, i.e., zebrafish in which gclc had been suppressed using CRISPR (sgRNA1+Cas9). (B') Representative brightfield image of the lateral view of $3.5 \mathrm{dpf}$ control and gclc KO (sgRNA1+Cas9) fish. Each point in $\mathbf{A}$ and $\mathbf{B}$ represents results from an individual zebrafish. Horizontal lines indicate the mean. * $\mathrm{P}<0.05$, one-way ANOVA with Tukey correction, compared to other group indicated. 